\title{
Gastrointestinal Symptoms and Outcomes in Hospitalized Coronavirus Disease 2019 Patients
}

\author{
Preethi Ramachandran ${ }^{a}$ Ifeanyichkwu Onukogu ${ }^{b}$ Snigdha Ghanta ${ }^{b}$ \\ Mahesh Gajendran $^{c}$ Abhilash Perisetti ${ }^{d}$ Hemant Goyal $^{\mathrm{e}}$ Alok Aggarwal $^{f}$ \\ aDepartment Hematology and Oncology, Brookdale University Hospital and Medical Center, Brooklyn, NY, USA; \\ bDepartment of Medicine, Brookdale University Hospital and Medical Center, Brooklyn, NY, USA; 'Department \\ of Medicine, Texas Tech University, Paul L. Foster School of Medicine, El Paso, TX, USA; dDepartment of \\ Gastroenterology and Hepatology, University of Arkansas for Medical Sciences, Little Rock, AR, USA; 'Department of \\ Medicine, The Wright Center for Graduate Medical Education, Scranton, PA, USA; 'Department of Hepatobiliary and \\ Pancreatic Surgery, Brookdale University Hospital and Medical Center, Brooklyn, NY, USA
}

\section{Keywords}

COVID-19 - Gastrointestinal manifestations ·

Gastrointestinal symptoms · Severe acute respiratory syndrome coronavirus 2 . Coronavirus - Outcomes .

Mortality $\cdot$ Length of stay

\footnotetext{
Abstract

Introduction: Gastrointestinal (GI) symptoms are increasingly being recognized in coronavirus disease 2019 (COVID-19). It is unclear if the presence of Gl symptoms is associated with poor outcomes in COVID-19. We aim to assess if GI symptoms could be used for prognostication in hospitalized patients with COVID-19. Methods: We retrospectively analyzed patients admitted to a tertiary medical center in Brooklyn, NY, from March 18, 2020, to March 31, 2020, with COVID-19. The patients' medical charts were reviewed for the presence of Gl symptoms at admission, including nausea, vomiting, diarrhea, and abdominal pain. COVID-19 patients with GI symptoms (cases) were compared with COVID-19 patients without Gl symptoms (control). Results: A total of 150 hospitalized COVID-19 patients were included, of which 31
}

(20.6\%) patients had at least 1 or more of the Gl symptoms (cases). They were compared with the 119 COVID-19 patients without $\mathrm{Gl}$ symptoms (controls). The average age among cases was 57.6 years (SD 17.2) and control was 63.3 years (SD 14.6). No statistically significant difference was noted in comorbidities and laboratory findings. The primary outcome was mortality, which did not differ between cases and controls ( 41.9 vs. $37.8 \%, p=0.68$ ). No statistically significant differences were noted in secondary outcomes, including the length of stay (LOS, 7.8 vs. 7.9 days, $p=0.87$ ) and need for mechanical ventilation ( 29 vs. $26.9 \%, p=0.82$ ). Discussion: In our study, the presence of Gl manifestations in COVID-19 at the time of admission was not associated with increased mortality, LOS, or mechanical ventilation.

(C) 2020 S. Karger AG, Basel

\section{Introduction}

The novel coronavirus disease 2019 (COVID-19) caused by severe acute respiratory syndrome coronavirus 2 (SARS-CoV-2) was started as an epidemic in Wuhan,

\section{KARGER}

(c) 2020 S. Karger AG, Basel

karger@karger.com

www.karger.com/ddi
Hemant Goyal

Department of Medicine, The Wright Center for Graduate Medical Education 501 S. Washington Avenue

Scranton, PA 18505 (USA)

doc.hemant@yahoo.com 
China. It was later declared as a global pandemic with New York as the current epicenter [1]. The SARS-CoV-2 mainly spreads through direct exposure (droplets, person to person). However, it is also assumed to be transmitted by contaminated objects, airborne transmission, and fecal-oral transmission [2]. The COVID-19 is predominantly a respiratory disease manifested by fever, fatigue, dry cough, anorexia, myalgia, and dyspnea [3]. However, gastrointestinal (GI) manifestations such as nausea, vomiting, diarrhea, and abdominal pain are increasingly being recognized as important manifestations of COVID-19 [4-8]. Other symptoms such as dysgeusia and anosmia are also gaining attention as important symptoms of COVID-19 $[9,10]$.

The spectrum of COVID-19 infection ranges from mild to critical. Most of the patients ( $81 \%$ ) have mild disease, $14 \%$ of the patients have severe disease, and $5 \%$ of the patients have a critical disease $[1,11]$. The factors associated with severe COVID-19 infection include advanced age $>65$ years, chronic respiratory diseases, hypertension, diabetes mellitus (DM), malignancy, and cardiovascular disease [12-14]. In a recent study published from the USA, about $12 \%$ of the patients required mechanical ventilation, and the mortality rate of patients on mechanical ventilation was $88 \%$ [15]. In a study by Pan et al. [7], the presence of GI symptoms was associated with higher liver enzymes, lower monocyte count, and longer prothrombin time. The overall pooled prevalence of GI symptoms in COVID-19 based on a systematic review was reported to be $18 \%$ [16]. The most common GI symptom reported is diarrhea (13\%), followed by nausea or vomiting (10\%) and abdominal pain [16]. SARS-CoV-2 has also been found in the fecal samples of COVID-19 patients even after the complete resolution of symptoms $[13,17]$. Therefore, the fecal-oral transmission is also considered as a potential mode of transmission [17]. The occurrence of GI symptoms is probably from the intestinal tropism of the SARS-CoV-2 [18]. Moreover, GI symptoms can coexist or even precede respiratory manifestations [19]. Rarely, COVID-19 patients can present with only GI symptoms without respiratory symptoms [7].

Hence, there has been an increasing interest in whether GI symptoms are associated with severe disease. There are conflicting reports in terms of whether GI symptoms are associated with severe COVID-19 or not [7, 20]. Therefore, in this study, we aimed to analyze if the presence of GI symptoms at the time of hospitalization is associated with mechanical ventilation or mortality when compared to those who did not have GI symptoms.
Table 1. Prevalence of gastrointestinal (GI) system in the entire cohort

\begin{tabular}{lc}
\hline GI symptoms & $N(\%)$ \\
\hline No gastrointestinal symptoms & $119(79.3)$ \\
Nausea/vomiting & $6(4)$ \\
Diarrhea & $15(10)$ \\
Nausea/vomiting + diarrhea & $7(4.7)$ \\
Nausea/vomiting + abdominal pain & $3(2)$ \\
\hline
\end{tabular}

\section{Methods}

Study Design and Data Source

This is a retrospective cohort study conducted in a cohort of COVID-19 patients who were admitted to the Brookdale University Hospital Medical Center (BHMCNY), a tertiary care academic medical center in Brooklyn, New York. BHMCNY is a non-profit medical service provider servicing almost 1 million residents of Eastern Brooklyn. The BHMCNY's Institutional Review Board approved this study as minimal-risk research while utilizing anonymized and de-identified retrospective data collection and waived the requirement for informed consent. We included consecutive patients who were admitted to the hospital with a confirmed diagnosis of COVID-19 on nasopharyngeal polymerase chain reaction testing for SARS-CoV-2 from March 18, 2020, to March 31, 2020. Patients were excluded if they were younger than 18 years, who were not hospitalized and managed on an ambulatory basis, pregnant patients, unavailability of results of SARS-CoV-2 nasopharyngeal testing, and missing data on mortality or disposition.

Data related to patients' demographics, clinical symptoms, comorbidities, home medications, vitals at presentation, admission laboratory tests, inpatient medications, and outcomes were collected (Table 1). Demographic variables such as age, sex, race, smoking status, and BMI were obtained. Data on multiple comorbid conditions such as the history of hypertension, dyslipidemia, coronary artery disease, DM, history of any cancer, chronic obstructive pulmonary disease, and asthma were obtained. Medication history of the use of angiotensin-converting enzyme inhibitor/angiotensin receptor blocker, non-steroidal anti-inflammatory drugs, aspirin, or statin use was collected. Furthermore, initial laboratory data such as hemoglobin level, WBC, absolute lymphocyte count, platelet count, ferritin, C-reactive protein (CRP), ddimer, lactic acid, aspartate aminotransferase, and alanine aminotransferase were noted. If a particular laboratory test was not performed at the time of admission, then the first laboratory values within $24 \mathrm{~h}$ of the admission were used.

\section{Stratification of Study Cohort and Outcomes}

In our study, the GI symptoms were defined as the presence of nausea, vomiting, diarrhea, or abdominal pain at the time of admission. The study cohort was stratified into 2 groups based on the presence of GI symptoms: COVID-19 with GI symptoms (cases) and COVID-19 without GI symptoms (controls). The primary outcome was death from any cause. Secondary outcomes were identified as total hospital length of stay (LOS) and need for mechanical ventilation during that hospitalization. 
Fig. 1. Study flowchart.

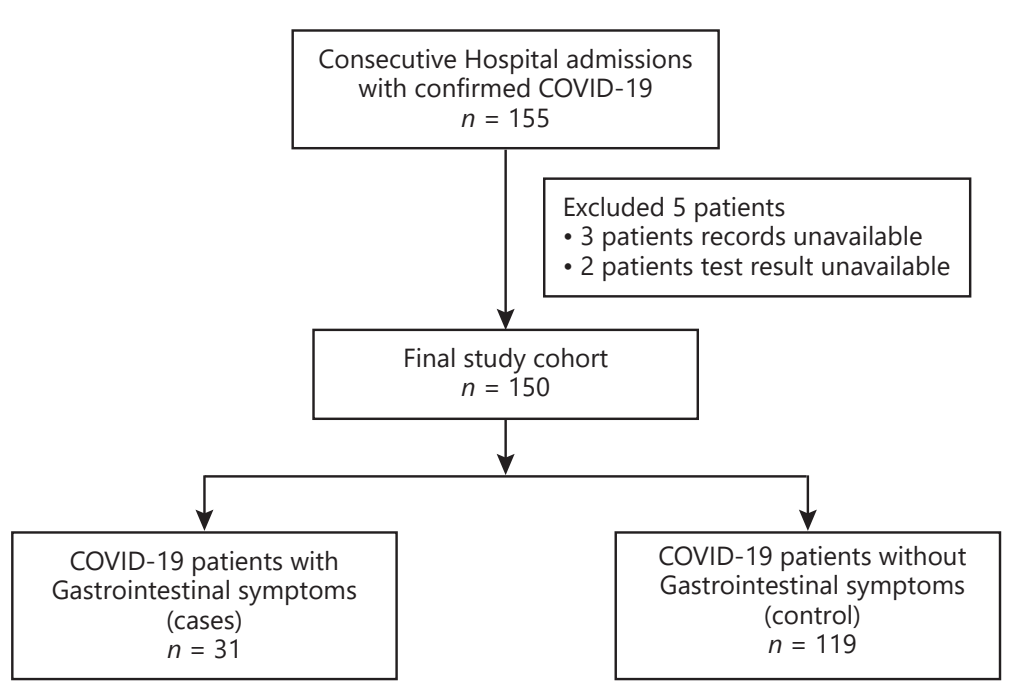

Table 2. Prevalence of individual gastrointestinal (GI) symptoms in the COVID-19 cohort with GI symptoms (cases)

\begin{tabular}{lc}
\hline GI symptoms & $N(\%)$ \\
\hline Nausea/vomiting & $6(19.4)$ \\
Diarrhea & $15(48.4)$ \\
Nausea/vomiting + diarrhea & $7(22.6)$ \\
Nausea/vomiting + abdominal pain & $3(9.7)$ \\
\hline
\end{tabular}

Statistical Analysis

Statistical analysis was performed using IBM SPSS software version 26 (SPSS Inc., Armonk, NY, USA). Descriptive summary statistics are presented as means and SD for continuous variables and frequencies with percentages for categorical variables. Categorical and continuous variables were tested for statistical significance using $\chi^{2}$ tests and $t$ tests, respectively. If the continuous variable is not normally distributed, we utilized the nonparametric test such as the Mann-Whitney $U$ test to compare the groups.

\section{Results}

\section{Study Population and Baseline Demographics}

A total of 155 patients were hospitalized with confirmed COVID-19 during the study period. Five patients were excluded based on the exclusion criteria. A total of 150 patients met the inclusion criteria and formed our final study population (Fig. 1). Of these, 31 (20.6\%) patients had GI symptoms (cases), and 119 patients had no GI symptoms (controls) (Table 1). Diarrhea was the most common GI symptom, which was reported in $14.7 \%$ of the cohort, followed by nausea or vomiting, reported in $10.7 \%$ of the patients, and only $2 \%$ of the patients had abdominal pain (Table 2). Demographic variables are noted in Table 3. The mean age was 57 years $(\mathrm{SD} \pm 17)$ in cases as compared to 63 years ( $\mathrm{SD} \pm 15$ years) in controls. The mean BMI was 31.7 and 30.7 in cases and controls, respectively. Comorbidities such as hypertension, dyslipidemia, chronic obstructive pulmonary disease, asthma, coronary artery disease, DM, and cancer were similarly distributed between 2 groups (Table 3 ). There was no difference in the presence of other symptoms such as fever, cough, dyspnea, fatigue, and myalgia between the 2 groups.

\section{Laboratory Data}

There was no statistical difference between the 2 groups in values of laboratory data such as mean hemoglobin, WBC, lymphocyte, and platelet counts. The mean ferritin level was lower in the cases than in controls but did not reach statistical significance (777 vs. $951 \mathrm{ng} / \mathrm{mL}$, $p=0.61$ ). Mean CRP, creatinine, and lactic acid levels were higher but not statistically significant in both groups, as noted in Table 4.

\section{Outcomes}

The outcomes of the study are outlined in Table 5. The patients with the GI symptoms (cases) had higher mortality of $41.9 \%$ (13/31 patients) when compared to controls, $37.8 \%$ (45/119 patients), but it did not reach statistical 
Table 3. Baseline demographics of the study population

\begin{tabular}{|c|c|c|c|}
\hline Characteristic & $\begin{array}{l}\text { COVID- } 19 \text { patients } \\
\text { with GI symptoms } \\
(N=31)\end{array}$ & $\begin{array}{l}\text { COVID-19 patients } \\
\text { without GI symptoms } \\
(N=119)\end{array}$ & $p$ value \\
\hline Age, mean (SD) & $57.6(17.2)$ & $63.3(14.6)$ & 0.06 \\
\hline Age $>60$ years, $n(\%)$ & $16(53.3)$ & $74(64.3)$ & 0.29 \\
\hline Female gender, $n(\%)$ & $12(38.7)$ & $55(46.2)$ & 0.54 \\
\hline BMI, mean (SD) & $31.7(8.8)$ & $30.7(7.6)$ & 0.57 \\
\hline \multicolumn{4}{|l|}{ Race, $n(\%)$} \\
\hline White & $3(9.7)$ & $3(2.5)$ & \\
\hline African American & $23(74.2)$ & $90(75.6)$ & \\
\hline Hispanic & $1(3.2)$ & $12(10.1)$ & 0.29 \\
\hline Asian & $1(3.2)$ & $6(5)$ & \\
\hline Unknown & $3(9.7)$ & $8(6.7)$ & \\
\hline \multicolumn{4}{|l|}{ Comorbidities, $n(\%)$} \\
\hline Hypertension & $22(14.7)$ & $79(66.4)$ & 0.67 \\
\hline Dyslipidemia & $10(32.3)$ & $44(37)$ & 0.68 \\
\hline CAD & $7(22.6)$ & $20(16.8)$ & 0.44 \\
\hline $\mathrm{DM}$ & $12(38.7)$ & $52(43.7)$ & 0.69 \\
\hline Cancer & $4(12.9)$ & $11(9.2)$ & 0.51 \\
\hline COPD & $1(3.2)$ & $13(10.9)$ & 0.30 \\
\hline Asthma & $6(19.4)$ & $17(14.4)$ & 0.58 \\
\hline Smoker & $4(12.9)$ & $13(10.9)$ & 0.75 \\
\hline \multicolumn{4}{|l|}{ Medications, $n(\%)$} \\
\hline ACEI/ARB & $11(35.5)$ & $38(31.9)$ & 0.83 \\
\hline NSAID & $6(19.4)$ & $28(23.5)$ & 0.81 \\
\hline Aspirin & $6(19.4)$ & $42(35.3)$ & 0.13 \\
\hline Statin & $12(38.7)$ & $58(48.7)$ & 0.42 \\
\hline \multicolumn{4}{|l|}{ Symptoms, $n(\%)$} \\
\hline Cough & $23(74.2)$ & $76(63.9)$ & 0.39 \\
\hline Fever & $22(71)$ & $79(66.4)$ & 0.67 \\
\hline Dyspnea & $17(54.8)$ & $82(68.9)$ & 0.20 \\
\hline Fatigue & $19(61.3)$ & $61(51.3)$ & 0.42 \\
\hline Myalgia & $12(38.7)$ & $47(39.5)$ & 1.00 \\
\hline Pneumonia & $29(93.5)$ & $119(100)$ & 0.04 \\
\hline
\end{tabular}

COVID-19, coronavirus disease 2019; GI, gastrointestinal; CAD, coronary artery disease; DM, diabetes mellitus; COPD, chronic obstructive pulmonary disease; ACEI/ARB, angiotensin-converting enzyme inhibitor/angiotensin receptor blocker; NSAID, non-steroidal anti-inflammatory drug.

significance $(p=0.68)$. No significant differences were noted in the secondary outcomes - mean LOS (7.8 [SD 4.4 ] vs. 7.9 days [SD 4.7 days], $p=0.87$ ) and need for mechanical ventilation ( 29 vs. $26.9 \%, p=0.82$ ).

\section{Discussion}

In this study, we found that $20.6 \%$ of the patients hospitalized with COVID-19 presented with at least 1 GI symptom such as diarrhea, nausea, vomiting, or abdominal pain. Diarrhea was the most common GI symptom, followed by nausea/vomiting and abdominal pain. There were no significant differences in terms of patient demographics, comorbid conditions, and presenting laboratory evaluations between patients with and without GI symptoms. Furthermore, there was no association between the GI symptoms and other symptoms such as fever, cough, fatigue, and myalgia.

Our study shows that the prevalence of GI symptoms in COVID-19 patients is $20.6 \%$, which is lower than the prevalence of GI symptoms reported by other studies in the USA and China in the range of $50.5-61.3 \%[7,21]$. The higher reported rate of GI symptoms in those studies could probably be due to the inclusion of anorexia as one of the GI symptoms. Anorexia is a nonspecific symptom 
Table 4. Laboratory data of both cohorts at the time of admission ${ }^{\mathrm{a}}$

\begin{tabular}{lccc}
\hline Laboratory test & $\begin{array}{c}\text { COVID-19 patients } \\
\text { with GI symptoms } \\
(N=31)\end{array}$ & $\begin{array}{l}\text { COVID-19 patients } \\
\text { without GI symptoms } \\
(N=119)\end{array}$ & $p$ value \\
\hline Hemoglobin & $12.6(2.2)$ & $13(1.8)$ & 0.28 \\
Ferritin & $776.9(961)$ & $951.8(1,253.1)$ & 0.61 \\
d-dimer & $901.6(1,380)$ & $10,661.9(22,910.3)$ & 0.36 \\
WBC & $7,200(2,100)$ & $7,400(3,700)$ & 0.78 \\
Lymphocyte count & $1,000(617)$ & $1,168(681.5)$ & 0.22 \\
Platelet count & $211,870(60,531)$ & $202,190(76,669)$ & 0.52 \\
Creatinine & $2.5(3.7)$ & $1.8(2.1)$ & 0.28 \\
Albumin & $3.9(0.5)$ & $3.7(0.5)$ & 0.07 \\
CPK & $391.8(602.8)$ & $1.99(1.7)$ & 0.48 \\
Lactate & $2.1(2.2)$ & $1,208.1(734.8)$ & 0.78 \\
LDH & $1,134.4(702.9)$ & $10.8(8.1)$ & 0.65 \\
CRP & $13.7(8.1)$ & $70.9(64.1)$ & 0.15 \\
AST & $71.4(77.3)$ & $51.8(44.4)$ & 0.97 \\
ALT & $52.1(63.3)$ & & 0.98 \\
\hline
\end{tabular}

COVID-19, coronavirus disease 2019; GI, gastrointestinal; CRP, C-reactive protein; AST, aspartate aminotransferase; ALT, alanine aminotransferase. ${ }^{a}$ All the laboratory values are represented as mean (SD).

Table 5. Outcome data of both cohorts at the time of admission

\begin{tabular}{llll}
\hline Characteristic & $\begin{array}{l}\text { COVID-19 patients } \\
\text { with GI symptoms } \\
(N=31)\end{array}$ & $\begin{array}{l}\text { COVID-19 patients } \\
\text { without GI symptoms } \\
(N=119)\end{array}$ & $p$ value \\
\hline Mortality, $n(\%)$ & $13(41.9)$ & $45(37.8)$ & 0.68 \\
Length of stay, mean (SD) & $7.8(4.4)$ & $7.9(4.7)$ & 0.87 \\
Mechanical ventilation, $n(\%)$ & $9(29)$ & $32(26.9)$ & 0.82 \\
\hline
\end{tabular}

COVID-19, coronavirus disease 2019; GI, gastrointestinal.

that could be related to an overall infectious or inflammatory process and hence was not included as a specific GI symptom in our study. In a US study by Redd et al. [20], the prevalence of GI symptoms in COVID-19 patients was reported to be as high as $61.3 \%$. In that study, anorexia was reported in $34.8 \%$ cases, diarrhea in $33.7 \%$, and nausea in $26.4 \%$ cases. In a study by Pan et al. [7] from Wuhan, China, even though about $50 \%$ reported having GI symptoms, the majority of these patients had anorexia $(78.6 \%)$. When anorexia was excluded from the analysis, only $18.6 \%$ had specific GI symptoms. A trend of increasing recognition of GI manifestations among COVID-19 patients is noted since its outbreak in Wuhan, China. During the original outbreak in Wuhan, diarrhea was reported in only $3 \%$ of the cases [1]. This number increased to $10 \%$ in a subsequent study from Wuhan and
$25 \%$ in a study from Singapore $[3,22]$. As awareness is increasing among the health-care workers about the GI manifestations in COVID-19 patients, the reports of the presence of GI symptoms increased in the studies.

In our study, there was no association between the GI symptoms and poor outcomes in COVID-19 patients. Previous studies have reported conflicting findings concerning the presence of GI symptoms and poor outcomes. In the study by Pan et al. [7] from Wuhan, China, patients with digestive symptoms had longer LOS (9 vs. 7.3 days, $p=0.013)$. Furthermore, this study noted that as the severity and duration of COVID-19 increase, GI symptoms increase as well. In a multicenter study of 191 patients by Zhou et al. [14], the presence of GI symptoms was associated with elevated CRP (7.3 vs. $3.8 \mathrm{mg} / \mathrm{L}, p=$ 0.021 ), elevated alanine aminotransferase (64.1 vs. 46.6 
units/L, $p=0.049$ ), and lower hemoglobin levels when compared to patients without GI symptoms. However, in the study by Redd and colleagues [20], there were no differences in clinical outcomes in patients with or without GI symptoms. Also, they reported no significant differences in the leukocyte count, hemoglobin, platelets, coagulation, or liver tests in groups with or without GI symptoms.

Although the specific mechanisms causing GI manifestations in COVID-19 are not entirely known, there are several proposed theories. Intestinal tropism has been noted with SARS-CoV-2, which could be due to its strong affinity to angiotensin-converting enzyme-2 receptors, and angiotensin-converting enzyme-2 receptors are highly expressed in the esophagus and intestinal epithelial cells [23]. Hence, there is a strong possibility of direct small bowel involvement, resulting in direct cytopathic effects causing GI symptoms. Furthermore, Redd et al. [20] noted that loss of smell (anosmia) and loss of taste (ageusia) were commonly associated with nausea (adjusted OR 2.71, 95\% CI: 1.21-6.20; $p=0.015$ ) and anorexia (adjusted OR 3.70, 95\% CI: 1.49-9.16; $p=0.0048$ ) after controlling for potential confounders. While the exact cause of this association is unclear, it could be due to damage to olfactory and gustatory receptors during viral entry through nasal and oral routes [24]. Additionally, in a study from Hong Kong, patients with diarrhea on presentation had higher rates of stool RNA positivity when compared to those without diarrhea ( 38.5 vs. $8.7 \%, p=0.02$ ). This is suggestive of the direct effects of the SARS-CoV-2 on the GI tract [16]. Also, the viral infection can cause altered intestinal permeability, resulting in malabsorption [25]. Finally, the inflammatory response from a cytokine storm in severe COVID-19 patients can cause hypoxia-induced bowel ischemia and contribute to diarrhea.

Specific limitations to this study include the retrospective design, relatively small sample, single-center hospital-based study, and lack of validated symptom instruments. This could introduce selection bias and limit the reliability and generalizability of the results. We could not correlate the presence of SARS-CoV-2 RNA with GI symptoms since this test was not routinely performed in our institution. Despite these limitations, the main strengths of this study are that it has validated the findings of another US study by Redd et al. [20] on GI manifestations in COVID-19. Our study also presents data from New York with a significantly higher proportion of African American patients. We also included data on home medications such as non-steroidal anti-inflammatory drugs and angiotensin-converting enzyme inhibitor/angiotensin receptor blocker, which has not been presented in previous studies.

\section{Conclusion}

GI symptoms are commonly encountered in hospitalized COVID-19 patients. In our study, GI symptoms were not associated with poorer outcomes such as increased mortality, longer hospital LOS, and increased mechanical intubation in COVID-19 patients. It appears that the GI symptoms could potentially be a bystander in patients with COVID-19. Further, more extensive studies are needed to evaluate the effects of GI symptoms on outcomes in COVID-19.

\section{Statement of Ethics}

The BHMCNY's Institutional Review Board approved this study as minimal-risk research while utilizing anonymized and deidentified retrospective data collection and waived the requirement for informed consent.

\section{Conflict of Interest Statement}

All authors have no conflicts of interest or financial ties to disclose.

\section{Funding Sources}

The authors did not receive any funding.

\section{Author Contributions}

P.R., A.P., and H.G.: Conception and design. P.R., I.O., and S.G.: Data collection. A.P. and M.G.: Drafting manuscript. P.R., I.O., S.G., M.G., A.A., H.G., and A.A.: Literature review, critical revision, and final approval of the manuscript.

\section{Availability of Data and Material} [26].

This own work has been deposited in a pre-print repository 


\section{References}

1 Huang C, Wang Y, Li X, Ren L, Zhao J, Hu Y, et al. Clinical features of patients infected with 2019 novel coronavirus in Wuhan, China. Lancet. 2020 Feb 15;395(10223):497-506.

2 Santarpia JL, Rivera DN, Herrera V, Morwitzer MJ, Creager H, Santarpia GW, et al. Transmission potential of SARS-CoV-2 in viral shedding observed at the University of $\mathrm{Ne}$ braska Medical Center.

3 Wang D, Hu B, Hu C, Zhu F, Liu X, Zhang J, et al. Clinical characteristics of 138 hospitalized patients with 2019 novel coronavirus-infected pneumonia in Wuhan, China. JAMA. 2020 Feb 7;323(11):1061-9.

4 Aloysius M, Thatti A, Gupta A. COVID-19 presenting as acute pancreatitis. Pancreatology. 2020;S1424-3903(20):30154.

5 Gao QY, Chen YX, Fang JY. 2019 novel coronavirus infection and gastrointestinal tract. J Dig Dis. 2020 Mar;21(3):125-6.

6 Kopel J, Perisetti A, Gajendran M, Boregowda $\mathrm{U}$, Goyal $\mathrm{H}$. Clinical insights into the gastrointestinal manifestations of COVID-19. Dig Dis Sci. 2020 Jul;65(7):1932-9.

7 Pan L, Mu M, Yang P, Sun Y, Wang R, Yan J, et al. Clinical characteristics of COVID-19 patients with digestive symptoms in Hubei, China: a descriptive, cross-sectional, multicenter study. Am J Gastroenterol. 2020 Apr 14; 115(5):766-73.

8 Perisetti A, Gajendran M, Goyal H. Putative mechanisms of diarrhea in COVID-19. Clin Gastroenterol Hepatol. 2020 Jun 11;S15423565(20):30708-1.

9 Aziz M, Perisetti A, Lee-Smith W, Gajendran $\mathrm{M}$, Bansal P, Goyal H. Taste changes (dysgeusia) in COVID-19: a systematic review and metaanalysis. Gastroenterology. 2020 May 5; S0016-5085(20):30595-3.
10 Giacomelli A, Pezzati L, Conti F, Bernacchia D, Siano M, Oreni L, et al. Self-reported olfactory and taste disorders in SARS-CoV-2 patients: a cross-sectional study. Clin Infect Dis. 2020 Mar 26:ciaa330.

11 Chen N, Zhou M, Dong X, Qu J, Gong F, Han $\mathrm{Y}$, et al. Epidemiological and clinical characteristics of 99 cases of 2019 novel coronavirus pneumonia in Wuhan, China: a descriptive study. Lancet. 2020 Feb 15;395(10223):50713.

12 Worldometer-age, sex, existing conditions of COVID-19 cases and deaths. https://www. worldometers.info/coronavirus/coronavirusage-sex-demographics/.

13 Perisetti A, Gajendran M, Boregowda U, Bansal P, Goyal H. COVID-19 and gastrointestinal endoscopies: current insights and emergent strategies. Dig Endosc. 2020 Apr 13.

14 Zhou F, Yu T, Du R, Fan G, Liu Y, Liu Z, et al. Clinical course and risk factors for mortality of adult inpatients with COVID-19 in Wuhan, China: a retrospective cohort study. Lancet. 2020 Mar 28;395(10229):1054-62.

15 Richardson S, Hirsch JS, Narasimhan M, Crawford JM, McGinn T, Davidson KW, et al. Presenting characteristics, comorbidities, and outcomes among 5700 patients hospitalized with COVID-19 in the New York city area. JAMA. 2020;323(20):2052-9.

16 Cheung KS, Hung IF, Chan PP, Lung KC, Tso E, Liu R, et al. Gastrointestinal manifestations of SARS-CoV-2 infection and virus load in $\mathrm{fe}$ cal samples from the Hong Kong cohort and systematic review and meta-analysis. Gastroenterology. 2020 Apr 3;S0016-5085(20): 30448-0.

17 Perisetti A, Garg S, Inamdar S, Tharian B. Role of face mask in preventing bacterial exposure to the endoscopist's face. Gastrointest Endosc. 2019 Nov;90(5):859.

18 Guan WJ, Ni ZY, Hu Y, Liang WH, Ou CQ, $\mathrm{He}$ JX, et al. Clinical characteristics of coronavirus disease 2019 in China. N Engl J Med. 2020 Apr 30;382(18):1708-20.
19 Liang W, Feng Z, Rao S, Xiao C, Xue X, Lin Z, et al. Diarrhoea may be underestimated: a missing link in 2019 novel coronavirus. Gut. 2020 Jun;69(6):1141-3.

20 Redd WD, Zhou JC, Hathorn KE, McCarty TR, Bazarbashi AN, Thompson CC, et al. Prevalence and characteristics of gastrointestinal symptoms in patients with SARS-CoV-2 infection in the United States: a multicenter cohort study. Gastroenterology. 2020 Apr 22; S0016-5085(20):30564-3.

21 Han C, Duan C, Zhang S, Spiegel B, Shi H, Wang $\mathrm{W}$, et al. Digestive symptoms in COVID-19 patients with mild disease severity: clinical presentation, stool viral RNA testing, and outcomes. Am J Gastroenterol. 2020 Jun; 115(6):916-23.

22 Young BE, Ong SWX, Kalimuddin S, Low JG, Tan SY, Loh J, et al. Epidemiologic features and clinical course of patients infected with SARS-CoV-2 in Singapore. JAMA. 2020 Mar 3;323(15):1488-94

23 Zou X, Chen K, Zou J, Han P, Hao J, Han Z. Single-cell RNA-seq data analysis on the receptor ACE2 expression reveals the potential risk of different human organs vulnerable to 2019-nCoV infection. Front Med. 2020 Apr; 14(2):185-92.

24 Vaira L, Salzano G, Deiana G, De Riu G. Anosmia and ageusia: common findings in COVID-19 patients. Laryngoscope. $2020 \mathrm{Jul}$; 130(7):1787.

$25 \mathrm{Gu}$ J, Han B, Wang J. COVID-19: gastrointestinal manifestations and potential fecal-oral transmission. Gastroenterology. 2020 Mar 3; 158(6):1518-9.

26 Ramachandran P, Onukogu I, Ghanta S, Gajendran M, Perisetti A, Goyal H, et al. Gastrointestinal Symptoms and outcomes in hospitalized COVID-19 patients. 